\title{
Quantitative High Performance Thin Layer Chromatography for the Analysis of Herbal Medicines: Problems and Advantages Snezana Agatonovic Kustrin* and Chandima G Hettiarachchi
}

\author{
School of Pharmacy and Applied Science, La Trobe University, Edwards Rd, Bendigo, 3550, Australia
}

Although medicinal plants have been used for thousands of years, there are still many issues associate with the quality control, standardization and effectiveness of herbal formulations [1]. Medicinal plants are complex mixtures with frequently unknown active constituents. They are mostly prepared from the crude extracts, not standardized or analysed for the content of the active ingredients. Quality control is difficult, as many factors can influence the final product; i.e. growth conditions of the plant, parts of the plant used, preparation of the plant for use, extraction method, volume of extract used in the final preparation and many others. All of these factors can affect the level of active compound, and therefore the effectiveness of the herbal formulation [2]. It is also difficult to define the active compound in medicinal plant since some constituents might demonstrate significant synergistic effect.

Adulteration and substitution of crude drugs with other plant material, is a major problem in herbal drug industry and is related to the safety issues. Adulterants are usually inferior commercial varieties and may or may not have any therapeutic potential as that of original drug. Adulteration can be intentional or unintentional and usually involves using plant material that is more readily available and similar in appearance to the intended medicinal variety. Intentional adulteration often occurs to enable cheaper production of by cutting the herbal material with a common plant species. This usually occurs because the medicinal plant can be difficult to obtain in large quantities [2]. Misidentification or confusion of common names may also lead to adulteration as seen in 1993 when a Belgium girl suffered renal failure after using Chinese herbal medicines for weight loss. In this case Stephania tetrandra was substituted with Aristolochia fangchi as they both had the common name of "fang ji" [3]. Similar confusion could potentially occur with C.officinalis as it has the common name "Marigold".

Tagetes erecta, known as "African Marigold" is a completely different species to C.officinalis and has been reported to be toxic [4]. This highlights the importance of the development of a quality control procedure to ensure the safety of herbal products. There are many steps involved in quality control of herbal medicines. The use of chromatography to develop fingerprints for specific plants allows many aspects of quality control to analysed simultaneously [5].

\section{Chromatographic Methods}

Several analytical techniques have been established for the identification and quantification of maker compounds in herbal formulations. Due to the complex nature of herbal formulations, chromatographic methods are commonly used to develop a fingerprint; a profile of the different constituents within the herbal product [6]. Fingerprinting can be used to identify the plant, quantify active ingredients or markers, and detect impurities or contaminants such as herbicides. Column chromatographic methods, such as High Performance Liquid Chromatography (HPLC) and Gas Chromatography (GC), are commonly used for fingerprinting [6,7]. However, HPLC is expensive to run, uses large quantities of solvent, and the column that is being used can be sensitive to very high or low $\mathrm{pH}$ mobile phases. GC is limited to the use of volatile compounds, such as essential oils in herbal preparations [6].
Due to its simplicity, speed, and relatively low cost, as number of samples can be analysed simultaneously on the same plate using a small amount of solvent as the mobile phase Thin Layer Chromatography (TLC) has been mostly used for the qualitative analysis of herbal medicines and to help characterise and track components visually or as an initial separation technique [8,9]. As it has been unable to provide accurate quantitative data TLC has become less favoured as quantification method of analysis $[6,10]$.

With advances in technology, thin layer chromatography has become more sophisticated and now has the potential to provide both qualitative and quantitative data [11]. High Performance Thin Layer Chromatography (HPTLC) plates provide has higher surface area and allows for quicker and clearer sample separation due to more consistent and significantly smaller particle size of the silica [12]. The use of HPTLC plates in combination with automated sample applicators and development chambers, high resolution cameras, and computing software allows more control over experimental conditions and greater data analysis capabilities [11,13]. HPTLC can run many samples simultaneously, uses small volumes of solvent, is cost effective, gives an instant visual result and is very user friendly compared to HPLC or GS $[13,14]$. These factors make it an effective analytical technique for quality control purposes particularly at the preliminary stage of testing and is already used extensively by the American Herbal Pharmacopoeia for identification [13].

A major advantage of HPTLC and TLC is the possibility of presenting the results as an image without the need for a specific detector, such as mass spectroscopy, and software. The different colours of the individual bands act as an extra dimension. Thus, in combination with visual evaluation HPTLC could provide extremely rapid screening of complex botanical samples. Compounds with native fluorescence are viewed as bright zones on a dark background under UV light. Substances without chromophores or colour that cannot be seen under UV-visible light can be visualized and made detectable by enhancing their fluorescence through post-chromatographic derivatization $[13,15]$. Derivatization is a technique used in TLC to change the properties of the sample by increasing detectability and/or visualization of the analyte. This can be performed on the plate immediately after the samples have been applied (pre-chromatographic dervatization) or after developing the plate. The derivatization reagent can be sprayed on to the plate or plate can be dipped into the derivatization reagent.

In HPTLC analysis inter-plate and inter-day variability are not

*Corresponding author: Snezana Agatonovic Kustrin, School of Pharmacy and Applied Science, La Trobe University, Edwards Rd, Bendigo, 3550, Australia, Tel: +61-3-5444-7360; Fax: +61-3-5444-7878; E-mail: s.kustrin@latrobe.edu.au

Received February 24, 2014; Accepted February 24, 2014; Published February 27,2014

Citation: Kustrin SA, Hettiarachchi CG (2014) Quantitative High Performance Thin Layer Chromatography for the Analysis of Herbal Medicines: Problems and Advantages. Mod Chem appl 2: e118. doi:10.4172/2329-6798.1000e118

Copyright: ( $) 2014$ Kustrin SA, et al. This is an open-access article distributed under the terms of the Creative Commons Attribution License, which permits unrestricted use, distribution, and reproduction in any medium, provided the original author and source are credited. 
uncommon. These differences occur due to the slight variances between plates and analytical conditions. These effects can be taken in to account by repeating the analysis several times on the same day and on different dates. Using an internal or external standard and adjusting the results accordingly is one way of minimizing the effects of inter-plate and interday variability. Although using an internal standard is more effective in minimising those effects, it may be difficult to find a suitable internal standard that is visible both before and after derivatization. In order to achieve this, an external standard must share certain characteristics with the active ingredients present in herbal preparation, but should not present in the herbal preparation that is analysed.

Most of the active constituents in herbal preparations are relatively unstable compounds which are prone to significant degradation when exposed to heat, light and air [16]. Due to the poor stability of the active ingredients, freshly prepared standard solutions had to be used to minimize errors in quantification. When analysing samples with low concentration of analytes such as herbal preparations, sample pre-concentration may be required to detect analytes. However, due to poor stability of the same, pre-concentration of samples can cause degradation of the active ingredients. Increasing the sample volume applied to the HPTLC plate is another way to increase the amount of the analyte, but it can lead to tailing of bands.

\section{Conclusion}

HPTLC chromatography may not have the same efficiency as HPLC separation but is simple to run, and multiple samples can be analysed and compared on the same plate. Furthermore, TLC is the only chromatographic method offering the choice of presenting the results as an image. Thus, in combination with visual evaluation, HPTLC could provide extremely rapid screening of investigated samples. The HPTLC method does not require sample preparation, analysis time is short and multiple samples can be analysed and compared simultaneously. Reliable quantitative results can also be obtained by HPLC, however in comparison with HPTLC, the procedure is solvent and time consuming and the sample preparation can be complicated.

\section{References}

1. (2004) The European Parliament and the Council of the European Union Directive 2004/24/EC of the European Parliament and of the Council of 31 March 2004 amending, as regards traditional herbal medicinal products, Directive 2001/83/EC on the Community code relating to medicinal products for human use. Official Journal of the European Union 136: 85-90.
2. Bandaranayake WM (2006) Quality control, screening, toxicity, and regulation of herbal drugs. Modern Phytomedicine: Turning Medicinal Plants into Drugs. 25-57.

3. Joshi VC1, Avula B, Khan IA (2008) Authentication of Stephania tetrandra S Moore (Fang $\mathrm{Ji}$ ) and differentiation of its common adulterants using microscopy and HPLC analysis. J Nat Med 62: 117-121.

4. Braun L (2005) Calendula- Calendula officinalis. Journal of Complementary Medicines 4: 71-81.

5. Blatter A, Reich E (2005) Qualitative and quantitative HPTLC methods for quality control of Stephania tetrandra. Journal of liquid chromatography \& related technologies 27: 2087-2100.

6. Tistaert C1, Dejaegher B, Vander Heyden Y (2011) Chromatographic separation techniques and data handling methods for herbal fingerprints: a review. Anal Chim Acta 690: 148-161.

7. Vander Heyden $Y$ (2008) Extracting information from chromatographic herbal fingerprints. LC-GC Europe 21: 438-443.

8. Gunther M, Schmidt PC (2005) Comparison between HPLC and HPTLC densitometry for the determination of harpagoside from Harpagophytum procumbens $\mathrm{CO}(2)$-extracts. J Pharm Biomed Anal 37: 817-821.

9. Fang C, Wan X, Jiang C, Cao H (2005) Comparison of HPTLC and HPLC for determination of isoflavonoids in several kudzu samples. Journal of Planar Chromatography - Modern TLC 18: 73-77.

10. Salmon CNA, Bailey-Shaw YA, Hibbert S, Green C, Smith AM, et al. (2012) Characterisation of cultivars of Jamaican ginger (Zingiber officinale Roscoe) by HPTLC and HPLC. Food chemistry 131: 1517-1522.

11. Houghton P, Mukherjee PK (2009) Evaluation of Herbal Medicinal Products: Perspectives on Quality, Safety and Efficacy. Pharmaceutical Press London, UK.

12. Spangenberg B, Poole C, Weins C (2011) Quantitative Thin-Layer Chromatography: A Practical Survey. Springer, Berlin, Germany.

13. Reich E, Schibli A (2006) High-performance thin-layer chromatography for the analysis of medicinal plants. Thieme Medical Publishers, New York, USA.

14. Dharmishtha M, Mishra SH, Falguni G (2009) Development and Validation of HPTLC Method for Quantitative Estimation of Oleanolic acid as Marker in Total Methanolic extract of Fruits of Randia dumetorum Lamk. Pharmacognosy magazine 5: 350-354.

15. Ling BL1, Baeyens WR, Del Castillo B, Stragier K, Marysael H, et al. (1989) Use of enhancers in the HPTLC fluorescence analysis of thiols. J Pharm Biomed Anal 7: 1671-1678.

16. Paixao N, Perestrelo R, Marques JC, Camara JS (2007) Relationship between antioxidant capacity and total phenolic content of red, rose and white wines. Food Chemistry 105: 204-214. 